\title{
THE EFFICIENCY OF THE PUMPING OF THE LASERS BASED ON SELF-TERMINATING ATOMIC TRANSITIONS OPERATING IN THE ENERGY INPUT CUT-OFF MODE
}

\author{
Alexey Burdin, Yury Polunin, Ivan Razmahnin, Anatoly Soldatov, Nikolay Yudin, \\ Nikolay Yudin* \\ National Research Tomsk State University, 634050, Tomsk, Russia
}

\begin{abstract}
The analysis of the electro-physical processes in the discharge circuit of the lasers based on the self-terminating transitions of metal atoms (LSTM) and the electrodes placed in the cold buffer zones of the gas discharge tube (GDT) is occurred. That design of the GDT can provide the efficient lasing at the reduction of the current flowing through the switch to zero after the charging of the capacitive components of the circuit from the storage capacitor. Under the circumstances the pumping of the active medium is determined by the energy input from the peaking capacitor and, consequently, the efficiency of the pumping can be increased by an order of magnitude, if (using a managed switch) the energy input into the active medium from the storage capacitor is "cut-off" after charging the capacitive components of the circuit. It was shown that the efficiency values of $\sim 9-11 \%$ and of $\sim 5-6 \%$ for the copper and gold vapor, lasers could be achieved.
\end{abstract}

\section{Introduction}

The lasers based on the self-terminating transitions of metal atoms (LSTM) are one of the most efficient coherent lasing sources in the visible spectrum among gas lasers and the predicted copper vapor laser efficiency is $\sim 10 \%$ [1], this is by an order of magnitude more than the achieved practical efficiency by now [2-4]. The highest value of the efficiency of the pumping of $\sim 9 \%$ of copper vapor laser operating in the energy input cut-off mode (the energy input into the active medium was excluded after a laser pulse) was achieved $[5,6]$. It can be explained by the fact that sharp-front pulses, which duration is equal to the lifetime of the population inversion of the operating levels in the active medium [1], should pump the LSTM. Due to the fact, that the duration of a pump pulse is higher than the lifetime of the population inversion of the operating levels in the active medium [2-4], the energy input cut-off after a lasing pulse is the obvious way to increase the efficiency of the pumping of the LSTM.

\footnotetext{
* Corresponding author: yudin@tic.tsu.ru
} 
In the experimental researches $[5,6]$ the managed switch consisting of the thyratron TGI1-270/12 and the tacitron TGU1-27/7 was used, what helped to realize the energy input cut-off mode at the circuit current of 27 A. According to the experimental researches $[5,6]$, the circuit current at the end of a laser pulse was higher than the mentioned value. Therefore, the tacitron should be close before the end of a laser pulse to realize the energy input cut-off mode. In this case, the duration of tacitron being open is equal to the thyratron jitter, that determines unstable operating, which was not observe during the experiments with the laser operating in the energy input cut-off mode. It is obvious that the modulation lamps GMI-29 [3] is the ideal switches for the lasers operating in the energy input cut-off mode. However, the efficiency of the pumping of the active medium of the LSTM with the modulation lamps that is equal to the value from $[5,6]$ was not realized. Thus, there are the several unanswered questions that limit using the energy input cut-off mode for the creating of the efficient sources of coherent light.

The purpose of the present work is the research of the electro-physical processes, in the discharge circuit of the laser, which determine the possibility of operating in the energy input cut-off mode.

\section{The electro-physical processes in the discharge circuit}

The population inversion of the LSTM is determined by the difference between the excitation rate of the upper and the lower operating laser levels by electron collisions during the non-equilibrium period of the plasma. The excitation rate constants of the laser levels of the LSTM are determined by the electron temperature, which depends on the voltage change on the active components of the active medium impedance [3]. Thus, the electro-physical processes in the discharge circuit of the LSTM determine the kinetic of the processes in the active medium. According to the electrical circuit theory, there are need to be created an equivalent circuit for the analysis of the electro-physical processes in the discharge circuit. The high pre-pulse concentration of electrons $\left(n_{\mathrm{e}} \sim 10^{13} \mathrm{~cm}^{-3}\right)$ in the active medium of the LSTM is typical. Thus, as suggested the gas discharge in the active medium occurs without the electrical breakdown and the equivalent circuit is the simple oscillatory circuit [3]. However, this equivalent circuit cannot explain the observed experimental dependencies, particularly the realizing of the efficient pumping of the active medium of the LSTM operating in the energy input cut-off mode $[5,6]$.

The presented in [7] equivalent circuit of the lasers based on the electrodes placed in the cold buffer zones of the gas discharge tube is more suitable for the explanation of the electro-physical processes in the discharge circuit. The mentioned design of the GDT is traditional for research and development of the LSTM [2-4]. In [5, 6] was studied the similar laser operating in the energy input cut-off mode. The pumping of the active medium occurs in two stages [7,8]. At the first (preparatory) stage, the charging of the capacitive components of the discharge circuit (peaking capacity $C_{0}$ and GDT's own capacity $C_{\mathrm{GDT}}$ ) by the energy stored in the storage capacity $C$ occurs. There are two modes of the charging of the capacitive components of the discharge circuit [7], which determine the population inversion, and these modes can be called — the modes of the "increased" and the "decreased" energy input. The existence of the two charge modes is determined by isolating the active medium, which geometric dimensions are govern by the heat-insulated discharge channel, from the electrodes by the cold buffer zones, in which there are no metal vapors. The charge neutralization during an inter-pulse period in the GDT, which has the mention design, occurs by the process of the three-particle recombination in the active medium and by the process of the dissociative recombination in the buffer zones [9]. Since the dissociative recombination rate is higher than the three-particle recombination rate [9], the charging of the capacitive components could occur at the total or partial recombination in 
the cold buffer zones. The mode change (from the "decreased" to the "increased" energy input) occurs with the increasing of the storage capacitor voltage [7] when the charging of the capacitive components (at the preparatory stage) of the discharge circuit from the storage capacitor at the partial recombination in the cold buffer zones occurs [7, 8]. Due to the increasing of the storage capacitor voltage results in the increasing of the energy output and the practical efficiency of the LSTM (relatively to the storage capacitor energy) [7], all research studied only the "increased" energy input mode and the "decreased" energy input mode was not studied.

The "decreased" energy input mode can be realized at the total recombination in the cold buffer zones and the decreasing of the current flowing through the switch to zero after the charging of the capacitive components from the storage capacitor, i.e. if the GTD voltage is equal to the breakdown voltage on the anode-side cold buffer zone of the GDT. After charging $C_{\mathrm{GDT}}$ during the breakdown process, according to $[7,8]$, the electric potential difference on the active medium occurs and (at the second stage) the pumping will be occurred during discharging $C$ and $C_{0}$. The edge condition of the start of the pumping of the active medium is $I_{1}=0$ and $I_{2}=0$, where $I_{1}$ and $I_{2}$ - the circuit current related to $C$ and $C_{0}$. Under these conditions the pumping of the active medium must be determined by the energy input from $C_{0}$, since the capacity value of the storage capacitor, ordinarily, is higher by an order of magnitude than the capacity value of $C_{0}$. That means that the efficiency of the pumping can be magnified by an order, if the switch like the tacitron excludes the energy input from the storage capacity into the active medium.

\section{The results of the experimental research and the discussion}

The research results of the electro-physical processes in the discharge circuit of the copper and gold vapor lasers illustrate all of the above. The experimental equipment circuit is presented in Figure 1.

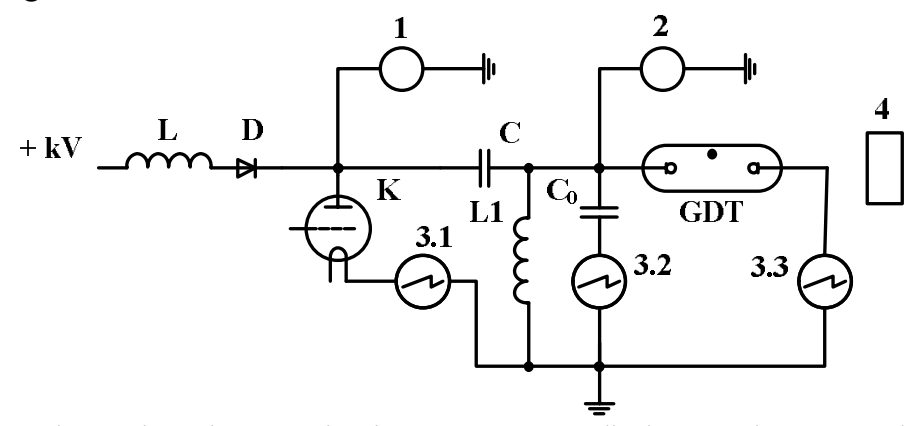

Fig. 1. The experimental equipment circuit: GDT - gas discharge tube; $\mathrm{K}$ - thyratron TGI11000/25; L, D - charging throttle and diode; L1 — shunt induction; C - storage capacitor; $\mathrm{C}$ - peaking capacity; 1 - static kilovoltmeter; 2 - voltage divider; 3 - current indicators; 4 - power meter.

The research was occurred with the heat-insulated GDT of the copper vapor laser at the pressure of the buffer gas $(\mathrm{Ne})$ of $\sim 80$ Torr. The GDT discharge channel is made of the $\mathrm{BeO}$-ceramics tube which the inner diameter of $8 \mathrm{~mm}$ and the length of $30 \mathrm{~cm}$. The capacitors KVI-3 was used as the storage and the peaking capacitors $\left(C=2200 \mathrm{pF}\right.$ and $C_{0}=$ $330 \mathrm{pF}$ ). The oscilloscope "Tektronix DPO-4034B" controlled the current and the voltage pulses. The average laser power was measured by the power meter "OPHIR-NOVA". The parameters (Figure 2) at the pulse repetition rate (PRR) of $10 \mathrm{kHz}$, the power consumption from the high-voltage rectifier of $\sim 350 \mathrm{~W}$ and the voltage $U_{\mathrm{r}}$ of $\sim 2.5 \mathrm{kV}$, the average laser power of $\sim 300 \mathrm{~mW}$ at the normal operating are presented. The pulses of the current 
flowing through the points (3.1), (3.2) and (3.3) (Figure 1) was measured by the same current sensor "Textronix" to exclude the measuring errors. The peak voltage on the storage capacitor of $U_{\mathrm{C}}=2 U_{\mathrm{r}} \sim 5 \mathrm{kV}$ under the said conditions of the pumping, i.e. at the matching mode of the electricity source with the electricity consumer, that did not allow to increase the voltage on the storage capacitor above $5 \mathrm{kV}$ because work of the thyratron is unsustainable. The typical CVL oscillograms of the pulses of the current flowing through the GDT 1, the voltage on GDT 2 and the lasing 3, are presented. In addition the oscillograms of the current 4 flowing through the thyratron during the discharge process of the storage capacitor and the (inverted) current 5 of the charging (5.1) and discharging (5.2) of $C_{0}$ are given. The current 4 flowing through the thyratron consists of two parts 4.1 and 4.2, where the first is the current of the charging of $C_{0}$ at the preparatory stage, the second is the current of the discharging of the storage capacitor at the pumping of the active medium. The identity of the pulses of the current 4.1 and 5.1 is the confirmation of the aforesaid. The invert oscillograms of the current 5 are presented (Figure 2) to demonstrate that the pulses 4.1 and 5.1 are identical.

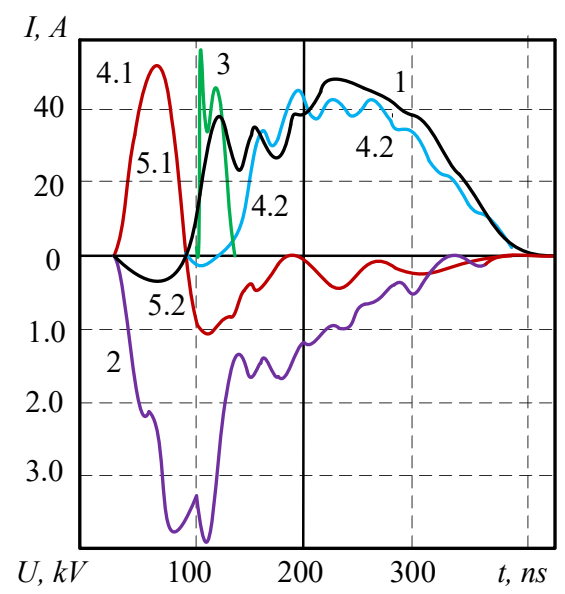

Fig. 2. The oscillograms of the pulses of the current (1) flowing through the GDT, the voltage (2) on GDT, the lasing (3), the current flowing through the thyratron (4) and the current (5) of the charging and the discharging of $C_{0}$. Where 4.1 is the current of the charging $C_{0}$ at the preparatory stage from the storage capacitor and 4.2 is the current of the discharging of the storage capacitor at the pumping, 5.1 is the current of the charging $C_{0}$ at the preparatory stage and 5.2 is the current of the discharging $C_{0}$ at the pumping.

The lasing (Figure 2) occurs during the discharging of $C_{0}$, and the energy input into the active medium from the storage capacitor occurs after the laser pulse and, accordingly, does not contribute in the population inversion generation. Thus at the excluding of the energy input into the active medium after the laser pulse in $[5,6]$ the changing of the position and the forms of the laser pulse did not occur. As follows from the presented results the excluding of the energy input into the active medium $[5,6]$ occurs before the laser pulse, i.e. after charging $C_{0}$, when the current flowing through the switch decreases to zero, that allows to close the tacitron.

The value of $C_{0}$, according to oscillograms (Figure 2), is $\sim 500 \mathrm{pF}$. The similar value of $C_{0} \sim 500 \mathrm{pF}$ can be calculated, according to the law of conservation of charge, under the mentioned conditions of the pumping, that less than the nominal value of $C_{0} \sim 330 \mathrm{pF}$ on $170 \mathrm{pF}$, and is determined by the value of the capacity of the conductive bus, that connected in parallel to $C_{0} \sim 330 \mathrm{pF}$. The efficiency of the laser relatively to the using energy from the rectifier under the mentioned conditions of the pumping is $\eta_{\mathrm{r}} \sim 0.09 \%$; relatively to the energy stored in the storage, capacitor is $\eta_{\mathrm{C}} \sim 0.11 \%$ and relatively to the energy stored in 
$C_{0}$ is $\eta \sim 0.75 \%$. These results confirm that the energy input cut-off mode results in the increasing of the efficiency of the pumping of the LSTM by an order of magnitude. Nevertheless, the obtained efficiency $(\eta \sim 0.75 \%)$ is less by an order of magnitude than the mentioned $(\eta \sim 9 \%)$ in [5]. The differences between the efficiency results are determined by the non-optimal value of the $C_{0} \sim 500 \mathrm{pF}$. To prove it purpose the GDT was connect to the storage capacitor and the shunt inductance (Figure 1) by the coaxial 2 meter in length cable. Under these conditions, the capacity of the coaxial cable act as $C_{0}$ and the conditions of the pumping can be optimized by changing of the length of the coaxial cable. The average laser power of the laser with the coaxial cable was $\sim 350 \mathrm{~mW}$ under the mentioned conditions of the pumping, and relatively to the energy stored in $C_{0} \eta \sim 2.2 \%$. The oscillograms of the pulses of the GDT voltage 2 and the lasing 3, the current 4 flowing through the switch during the charging of the storage capacitor and (inverted) the charge (5.1) and discharge (5.2) current 5 with the coaxial 2 meter in length cable are presented on Figure 3.

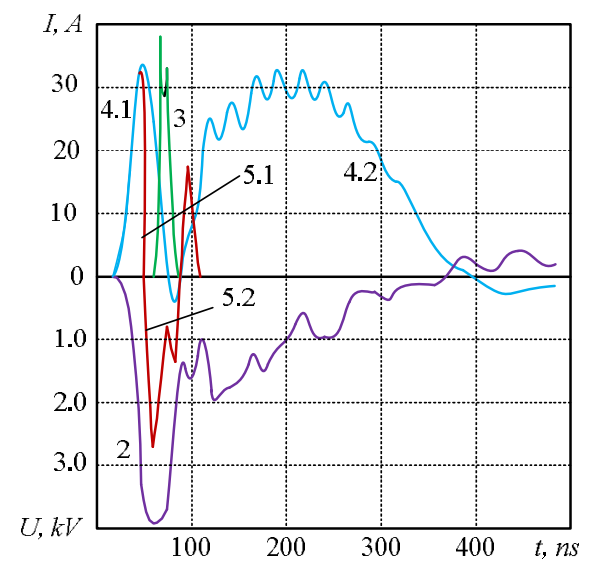

Fig. 3. The oscillograms of the pulses of the GTD voltage (2), the lasing (3), the current flowing through the switch (4) and the charge and discharge current (5) of $C_{0}$ (the coaxial $2 \mathrm{~m}$ in length cable). 4.1 - the charge current of $C_{0}$ from the storage capacitor at the preparatory stage and 4.2 - the discharge current of the storage capacitor at the pumping, 5.1 - the charge current of $C_{0}$ at the preparatory stage and 5.2 - the discharge current of $C_{0}$ at the pumping.

Under the similar conditions of the pumping using the coaxial $\sim 1 \mathrm{~m}$ in length cable the efficiency of $\eta \sim 2.8 \%$ was occurred. This mode can be obtained at the less than $17 \mathrm{kHz}$ PRR. The storage capacitor of $\sim 1320 \mathrm{pF}$ connected to the GDT through the coaxial $1 \mathrm{~m}$ in length cable was used for obtaining the self-heating mode at the $17 \mathrm{kHz}$ PRR.

The further studies were carried out with the GDT of the gold vapor laser at the pressure of the buffer gas $(\mathrm{Ne})$ of $\sim 60$ Torr. The discharge channel of the GDT is made of BeOceramic tube of $10 \mathrm{~mm}$ in inner diameter and $50 \mathrm{~cm}$ in length. The capacitor KVI-3 $(C=$ $3300 \mathrm{pF}$ ) was used as the storage capacitor. The energy characteristics of lasing and the electro-physical parameters were measured at the PRR of the pumping of $12.5 \mathrm{kHz}$, the power consumption of the rectifier of $\sim 1.2 \mathrm{~kW}$ and the voltage of $U_{\mathrm{r}} \sim 2.8 \mathrm{kV}$ after the stabilization of the laser operating. The dependencies of the average laser power $(\lambda=627.7 \mathrm{~nm})$ from the value of the peaking capacity and the efficiency of the pumping of the active medium from the energy stored in the peaking capacitor are presented on Figure 4. Furthermore the increasing of the average laser power (Figure 4, graph 2) after putting the induction $L_{\mathrm{d}}$ into the discharge circuit between the storage capacitor and the peaking capacitor $(330 \mathrm{pF})$ was observed, due to there was the resonance charging of the peaking capacitor and the storage capacitor. 
The energy parameters of the copper and gold vapor lasers was measured at the decreasing to zero of the current flowing through the switch after the charging of the capacitive components of the circuit (Figure 1 and Figure 2). However, the increasing of the peaking capacitor $\left(C_{0} \sim 220 \mathrm{pF}\right)$ voltage of $\sim 8 \%$ (the energy stored in the peaking capacitor of $\sim 17 \%$ ) results in the increasing of the average laser power by an factor of 2-3 times at the minimal value of the current flowing through the switch of $\sim 20 \mathrm{~A}$ after the charging of the capacitive components of the circuit. It is obvious that these values of the current flowing through the switch allow the laser to operate in the energy input cut-off mode. Consequently, the efficiency of $\sim 9-11 \%$ and of $\sim 5-6 \%$ for the copper and gold vapor lasers operating in the energy input cut-off mode could be achieved.

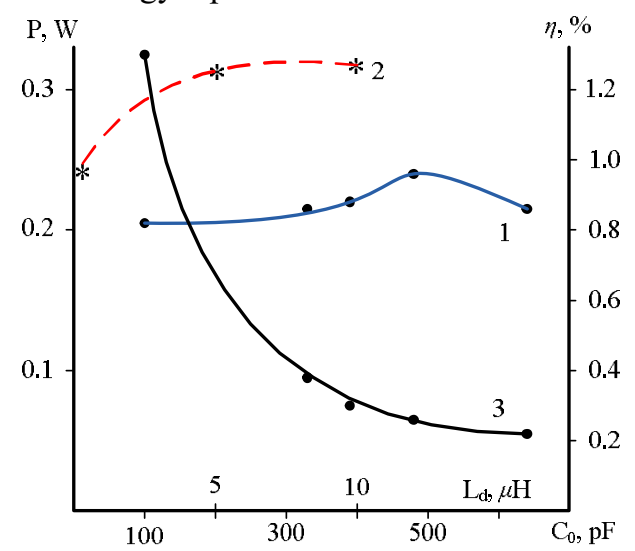

Fig. 4. The dependencies of the average laser power $(\lambda=627.7 \mathrm{~nm})$ from the value of the peaking capacity -1 , the value of the inductance putted between the storage capacitor and the peaking capacitor -2 and the efficiency of the pumping of the active medium from the energy stored in the peaking capacitor -3 .

There are need to be mentioned, that the energetic parameters of the copper and gold vapor lasers were measured at the source and load matching, that means the condition of the aperiodic discharge of the storage capacitor was fulfilled. Also the mismatch relatively to the energy input from the peaking capacitor was observed, that grows at the decreasing of the value of the peaking capacity in the copper and gold vapor lasers (Figure 2 (5.1)(5.2) and Figure 3 (5.1)-(5.2), Figure 5 (1)). Higher mismatching was observed in gold vapor laser (Figure 5).
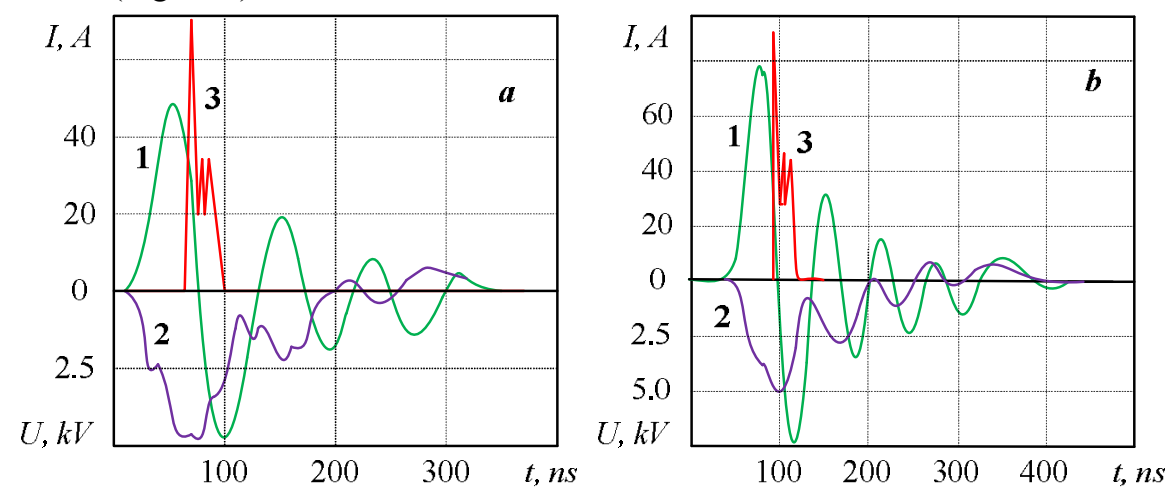

Fig. 5. The oscillograms of the pulses of the GDT voltage (2), the lasing (3) and the charge and discharge current (1) of the $C_{0}$ of the gold vapor laser: a) $C_{0}=330 \mathrm{pF}$, b) $C_{0}=220 \mathrm{pF}$. 
The occurred researches confirm the possibility of the efficient pumping of the active medium of the LSTM and allow explain, according to the equivalent circuit [7], the causes of the decreasing of the efficient pumping at the growth of the storage capacitor voltage. This decreasing is explained by the fact that the capacitive components of the circuit from the storage capacitor (at the preparatory stage) are charged at the shunting of the capacitive components of the anode-side cold buffer zone of the GDT and the overrating of the voltage of the breakdown voltage $U_{\mathrm{br}}$ of the cold buffer zones. This determines the fact of the increasing of the amplitude of the GDT voltage $U_{\mathrm{a}}$ till the specified value $U_{\max }$ at the increasing of the storage capacitor voltage $U_{\mathrm{C}}$. The specified value $U_{\max }$ is determined by the charge input rate $v_{1}$ (the rate of the increasing of the current flowing through the switch) from the storage capacitor into the capacitive components of the circuit and the charge output rate $v_{2}\left(v_{2}=f\left(U_{\mathrm{a}}\right)\right)$ to anode of the GDT. It is obvious that the increasing of the voltage amplitude $U_{\mathrm{a}}$ is observed at $v_{1}>v_{2}$ till value $U_{\max }$ at $v_{1}=v_{2}$ and determines the optimal storage capacitor voltage $U_{\text {opt }}$ at the specified experimental conditions, that allow obtain the most efficient pumping relatively to the energy stored in the storage capacitor. The charging of the capacitive components of the circuit from the storage capacitor occurs till the capacitive components $\left(C, C_{0}\right.$ and $\left.C_{\mathrm{GDT}}\right)$ voltage equalization, when $U_{\mathrm{C}} \sim U_{\max }$. The further process is accompanied by the decreasing of the current flowing through the switch and the condition of $v_{2}>v_{1}$ is fulfilled that determines the discharging of the GDT capacity. These processes are crucial for the population inversion generating in the active medium, due to during this period the features of the mentioned tube design better appear. The high-conductive $\left(n_{\mathrm{e}} \sim 10^{13} \mathrm{~cm}^{-3}\right)$ active medium, the discharge ceramic channel and the reverse current wire constructively are the capacitor $C_{\mathrm{GDT}}$, one plate of which is the active medium. The feature of this is that the plasma, according to the definition, is quasineutral and an excess charge cannot accumulate in the plasma during the charging of the capacitive components. Consequently, an excess charge should be placed on the plasma surface, i.e. at the inner wall of the discharge ceramic channel. The potential difference between the plates of the capacitor occurs during $C_{\mathrm{GDT}}$ charging, but the plasma is still under the same potential, that prevents the pumping of the active medium during this period, that confirm the experimental research [10]. The charge transfer to the anode during the charging of the capacitive components determines the phantom current $I_{\mathrm{ph}}$, which maximum value can be $60-70 \%$ relatively to the peak value of the circuit current. [7, 10]. The $C_{\mathrm{GDT}}$ charging occurs after the charging of the capacitive components from the storage capacitor discharging, the potential difference on the active medium occurs, that determines the pumping process. This process, called in [10] the "breakdown" and in [8] the "pseudobreakdown", is determined by the fact that the discharging of $C_{\mathrm{GDT}}$ occurs not through the active medium and can be considered as the diffusion of electrons through the surface of the discharge ceramic channel to the anode through the cold buffer zone [11], that determines the sharp front of the pulse of the pumping. Wherein the significant share of the energy $E_{\mathrm{L}}=L\left(I_{\mathrm{ph}}\right)^{2} / 2$ accumulated in the inductance $L$ almost does not contribute to the population inversion generation, due to the period of the discharging of the storage inductance into the active electrical consumer is $\sim L / R$ and, consequently, the storage inductance is ineffective at the typical of LSTM the high plasma conduction.

The energy consumption in the mentioned processes was studied in [10]. The inductance of the discharge circuit of the laser, according to the oscillograms from [10], of $\sim 1.1 \cdot 10^{-6} \mathrm{H}$. The GDT voltage amplitude is $\sim 10 \mathrm{kV}$ and the phantom current amplitude is $\sim 600$ A. The energy stored in the peaking capacitor at the moment of the breakdown under these conditions is $E_{\mathrm{C} 0} \sim 0.16 \mathrm{~J}$, in the circuit inductance is $E_{\mathrm{L}} \sim 0.19 \mathrm{~J}$ and in the storage capacitor is $E_{\mathrm{C}}{ }^{\mathrm{b}} \sim 0.35 \mathrm{~J}$. Due to the fact that in [10] the full set of the necessary information for the energy consumption calculating was not presented the further calculations will be based on the indirect estimates. The estimation $C_{\mathrm{GDT}}$ of $\sim 0.6 \mathrm{nF}$ was 
based on the fact that the condition $E_{\mathrm{C} 0}+E_{\mathrm{GDT}}=E_{\mathrm{L}}$ should be fulfilled at the moment of the breakdown. The total energy stored in the reactive components of the circuit $\left(E_{\mathrm{C}}^{\mathrm{b}}+E_{\mathrm{C} 0}+\right.$ $E_{\mathrm{GDT}}+E_{\mathrm{L}}$ ) is $\sim 60 \%$ relatively to the energy initially stored in the storage capacitor, what determines the significant energy consumption of the charge displacement operation during the charge process of the circuit capacitive components from the storage capacitor and determines the high initial energy losses in the switch [3]. Consequently, the consumed power by the laser should be $\sim 8 \mathrm{~kW}$ at the PRR of the lasing of $6.5 \mathrm{kHz}$, and the efficiency of $\sim 0.5 \%$ at the average laser power of $\sim 40 \mathrm{~W}$ or $3.3 \%$ relatively to the energy $\left(E_{\mathrm{C} 0}+\right.$ $E_{\mathrm{GDT}}$ ), that determines the pumping of the active medium.

The mentioned above estimates explain the causes of the insignificant increasing of the efficiency of the pumping of the LSTM using the modulation lamps, like GMI-29, as the switch [3]. In this case, there is the decreasing of the energy losses (if the energy input is cut-off at the moment of the breakdown) by the value $E_{\mathrm{C}}{ }^{\mathrm{b}}$ or $\sim 30 \%$ relatively to the energy stored in the storage capacitor. In fact, it is impossible to technically realize the excluding of the energy input into the active medium at the moment of the breakdown using the modern switches, because in this case there should be the voltage $U=-L(d I / d t)$ that is determined by the current break that significant exceeds the possible voltage value of the switch. For this reason, the pumping of the active medium of the LSTM occurs using the modulation lamps connected with the GDT from the storage capacitor through the $10 \mathrm{~m}$ in length cable acting as the long line and the peaking capacitor $C_{0}$. The charging of the long line occurs from the storage capacitor when the lamp opens for $t \leq 50 \mathrm{~ns}$. Then the charging of $C_{\mathrm{GDT}}$ and $C_{0}$ occurs and the further processes are identical to the mentioned above.

\section{Conclusion}

The carried out researches confirm the possibility of the gas discharge pumping of the LSTM with the predicted efficiency and allow evaluate the technical solutions operating in the "increased" energy input mode. The general approach to solving of this problem - is the resonance charging of the circuit capacitive components with the further discharging of them into the active medium, what can be done, at least, by the two ways. The first was studied in [12] where the discharging of the storage capacitor by the high-rate switch through the active medium of the GDT, which electrodes are placed in the hot zone of the discharge channel, was proposed. The second is suitable for the pumping of the active medium of the GDT, which electrodes are placed in the cold buffer zone. This approach of the pumping can be realized using the managed switch that should be closed after the resonance charging (by the putting of the additional inductance into the circuit between the storage capacitor and the peaking capacitor) $C_{0}$ and $C_{\mathrm{GDT}}$ from the storage capacitor. For realizing this condition there are need to be the additional switch putted in the anode-side zone of the circuit, which should be opened after charging $C_{0}$ and $C_{\mathrm{GDT}}$, when the current flowing through the switch decreases to zero. This condition can be realized using the kiwotron $[13,14]$ as the additional switch.

The carried out researches demonstrate the possibility of the increasing of the efficiency of the pumping of the active medium of the LSTM due to the decreasing of the energy consumption of the population inversion generation. However, the energy consumption of the population inversion generation is not the main factor that determines the energy characteristics of the LSTM. Now it is well known, that the main cause of the limiting of the output characteristics of the LSTM is the high rate of the de-excitation of the laser levels by the stepwise ionization [15-17], that limits the population of the resonance levels [18] and, consequently, the energy output [16]. The population inversion value under these conditions is totally determined by the processes of the excitation the lower laser levels during the front pulse of the pumping and by the processes of their de-excitation during the 
inter-pulse period, and the increasing of the average laser power is possible due to the increasing of the pulse repetition rate (PRR) of the lasing. Consequently, the energy output is determined by the electro-physical process in the discharge circuit of the LSTM, which determines the need for further research. The interpretation difficulty of the research results of the electro-physical processes in the discharge circuit of the laser is determined by the fact that the electrical circuit includes plasma object, in which the processes were not studied until now, particularly, the processes in the cold buffer zones. That determines the need for the further detail research of the electro-physics and the physics of the pulse period gas charge. However, the carrying out this research is expediently if there is the potential possibility of the increasing of the frequency-energy characteristics of the LSTM. The mentioned above estimates, which demonstrate that the energy consumption of the population inversion generation in the laser [10] at the energy output of $\sim 3-4 \mu \mathrm{J} / \mathrm{cm}^{-3}$ can be decreased by a factor of 4 without changing the parameters, determine the continuation of the research. Thus, there is the potential possibility of the increasing of the PRR of the lasing to $\sim 26 \mathrm{kHz}$ and the average laser power to $\sim 160 \mathrm{~W}$, what confirms the research results [14]. Wherein the average laser power can be increased due to the optimization of the parameters of the pumping to $\sim 500-800 \mathrm{~W}$, because the energy output value can be $20 \mu \mathrm{J} / \mathrm{cm}^{-3}$ at the GDT voltage of $10 \mathrm{kV}$.

\section{References}

[1] G.G. Petrash, Sov. Physics - Uspekhi 14, 747 (1972)

[2] A.N. Soldatov, V.I. Solomonov, Gas-discharge lasers on self-limited transitions in metal vapor (Science, Novosibirsk, 1985)

[3] V.M. Batenin, V.V. Buchanov, M.A. Kazaryan, I.I. Klimovsky, E.I. Molodykh, Lasers on self-limited transitions of metal atoms (Scientific book, Moscow, 1998)

[4] C.E. Litlle, Metal Vapour Lasers. Physics, Engineering and Application (John Wiley \& Sons, New York, 1999)

[5] A.N. Soldatov, V.F. Fedorov, N.A. Yudin, Quantum Electronics 24, 677 (1994)

[6] A.N. Soldatov, N.A. Yudin, J. of Russian Laser Research 16, 128 (1995)

[7] N.A. Yudin, V.B. Sukhanov, F.A. Gubarev, G.S. Evtushenko, Quantum Electronics, 38, 23 (2008)

[8] N.A. Yudin, N.N. Yudin, Russian Physics Journal 58, 1782 (2016)

[9] K.I. Zemskov, A.A. Isaev, G.G.Petrash, Quantum Electronics 29, 462 (1999)

[10] G.P. Hogan, C.E. Webb, Optics Communication 117, 570 (1995)

[11] Yu. Akishev, G. Aponin, A. Balakirev, et al., J. Phys. D: Appl. Phys. 46, 464014 (2013)

[12] N.A. Yudin, N.N. Yudin, Russian Physics Journal 59, 809 (2016)

[13] P.A. Bokhan, P.P. Gugin, Dm. E. Zakrevsky, M.A. Lavrukhin, Technical physics letters 38, 383 (2012)

[14] P.A Bokhan., P.P. Gugin, Dm. E. Zakrevsky, M.A. Lavrukhin, M.A. Kazaryan, N.A. Lyabin, Quantum Electronics 43, 715 (2013)

[15] A.V. Eletsky, Yu. K. Zemtsov, A.B. Rodin, A.I. Starostin, Reports of the USSR Academy of Sciences 220, 318 (1975)

[16] Yu. A. Piotrovsky, N.M. Reutova, Yu. A. Tolmachev, Optics and Spectroscopy 7, $99(1984)$

[17] N.A. Yudin, V.M. Klimkin, V.E. Prokopyev, Quantum Electronics 29, 828 (1999)

[18] A.A. Isaev, V.T. Mihkelsoo, G.G. Petrash, B.E. Peet, I.V. Ponamorev, A.B. Treshchalov, Sov. J. Quantum Electronics 18, 1577 (1988) 